\title{
Low-dose Amiodarone Is Safe: A Systematic Review and Meta-analysis
}

\author{
RONPICHAI CHOKESUWATTANASKUL, MD, ${ }^{1,2}$ NUPUR SHAH, MD, ${ }^{3}$ SUSAMA \\ CHOKESUWATTANASKUL, MD, ${ }^{4}$ ZHIGANG LIU, MD, ${ }^{5}$ and RANJAN THAKUR, MD, MPH, FHRS ${ }^{1}$
}

\author{
'Sparrow Hospital, Michigan State University, Lansing, MI, USA \\ ${ }^{2}$ Faculty of Medicine, King Chulalongkorn Memorial Hospital, Chulalongkorn University, Bangkok, Thailand \\ ${ }^{3}$ St. Mary Mercy Hospital, Livonia, MI, USA \\ ${ }^{4}$ Department of Ophthalmology, Faculty of Medicine, Chiang Mai University, Chiang Mai, Thailand \\ ${ }^{5}$ Division of Cardiac Electrophysiology, University of Michigan Health Care, Ann Arbor, MI, USA
}

\begin{abstract}
Amiodarone is commonly used for a variety of arrhythmias and, in some parts of the world, is the only available antiarrhythmic drug $(A A D)$. Yet, amiodarone is known to have a wide range of potential side effects, many of which are dose- and duration-dependent. We sought to study the incidence of side effects leading to the discontinuation of low-dose amiodarone, arbitrarily defined as $200 \mathrm{mg}$ /day or less, and very-low-dose amiodarone, defined as $100 \mathrm{mg} /$ day or less. In this study, literature databases were searched through June 2019. Studies that reported the incidence or prevalence of side effects of amiodarone were included. Effect estimates from individual studies were extracted and combined using the random-effects generic inverse variance method of DerSimonian and Laird. A total of 10 observational cohort studies involving 901 patients were included in the analysis. The pooled estimated incidence of overall side effects for low-dose amiodarone was 0.17 [95\% confidence interval (CI): 0.12-0.22]. In addition, the pooled estimated incidence of side effects requiring medication discontinuation was 0.06 (95\% CI: 0.03-0.11). As compared with $200 \mathrm{mg} /$ day of amiodarone, the pooled estimated incidence of overall side effects was 0.11 (95\% CI: 0.04-0.27), while the incidence of side effects requiring medication discontinuation was 0.02 (95\% CI: 0.01-0.06) for the dose of $100 \mathrm{mg} /$ day. In conclusion, verylow-dose amiodarone displays a low incidence of significant side effects requiring medication discontinuation.
\end{abstract}

KEYWORDS. Amiodarone, low dose, meta-analysis, safety, side effect.
ISSN 2156-3977 (print) ISSN 2156-3993 (online) CC BY 4.0 license

C 2020 Innovations in Cardiac Rhythm Management

\section{Introduction}

Amiodarone is commonly utilized for treating both supraventricular and ventricular arrhythmias. While this drug is a very effective antiarrhythmic agent, it also leads to many well-known side effects involving a variety of organs such as the thyroid, liver, lungs, and eyes including many that are dose- and duration-dependent. ${ }^{1}$

The authors report no conflicts of interest for the published content. Manuscript received September 13, 2019. Final version accepted October 7, 2019.

Address correspondence to: Ranjan K. Thakur, MD, Sparrow Thoracic and Cardiovascular Institute, 1440 East Michigan Avenue, Suite 400, Lansing, MI 49912, USA. Email: thakur@msu.edu.
Therefore, the use of amiodarone must be balanced between the drug's potentially serious adverse effects and its antiarrhythmic effects. However, current guidelines still recommend that amiodarone be chosen as the first-line therapy in some patient groups. ${ }^{2}$

Some known adverse effects of amiodarone may be related to the dose and duration. In 1997, a meta-analysis of low-dose amiodarone, defined as less than $400 \mathrm{mg} /$ day, reported a higher rate of drug discontinuation as compared to placebo $(22.9 \%$ versus $15.4 \%){ }^{3}$ With the development of new therapeutic agents, catheter ablation, and implantable cardioverter-defibrillators (ICDs) for the management of arrhythmias, a lower dose of 
amiodarone is often sufficient, and studies have shown a degree of clinical efficacy in using a low dose to suppress arrhythmias. ${ }^{4,5}$ A survey in Europe also suggested that very-low-dose amiodarone, defined as $100 \mathrm{mg} /$ day or less, is commonly used to treat arrhythmia. ${ }^{6}$ However, there are no well-designed randomized studies examining the efficacy and safety of very-low-dose amiodarone. Still, many observational studies show promising findings in terms of the safety of very-lowdose amiodarone. ${ }^{4,7}$

Given the above, we conducted the present systematic review and meta-analysis to assess the incidence, prevalence, and odds of side effects of low-dose $(\leq 200 \mathrm{mg} /$ day) and very-low-dose ( $\leq 100 \mathrm{mg} /$ day) amiodarone treatment regardless of arrhythmia indication.

\section{Methods}

\section{Literature review and search strategy}

The protocol for this meta-analysis was registered with the International Prospective Register of Systematic Reviews (no. CRD42018089481). A systematic literature search of MEDLINE (1946 to March 2019), EMBASE (1988 to March 2019), and the Cochrane Database of Systematic Reviews (database inception to March 2019) was conducted to identify studies evaluating associations of amiodarone and side effects in patient with all types of arrhythmias. The systematic literature review was undertaken independently by two investigators (R. C. and R. K. T.), applying a search approach that incorporated variations of "amiodarone" or "side effect" and "safety," which are covered in Table 1. No language limitation was applied. A manual search for conceivably relevant studies among the references of the included articles was also performed. This study was conducted according to the Preferred Reporting Items for Systematic Reviews and Meta-Analysis statement.

\section{Selection criteria}

Eligible studies included cross-sectional, case-control, or cohort studies that assessed the associations of side effect and safety and provided the effect estimates of incidence, prevalence, odds ratios (OR), relative risks (RR), or hazard ratios (HR) with 95\% confidence intervals (CIs). Study size did not dictate inclusion status. Retrieved articles were individually reviewed for their eligibility by the two investigators noted previously. Discrepancies

Table 1: Search Terms Applied in the MEDLINE, EMBASE, and Cochrane Databases

\begin{tabular}{|l|}
\hline 1. exp amiodarone/ \\
2. amiodarone $\$ . m p$ \\
3. 1 or 2 \\
4. exp side effect/ \\
5. adverse $\$ . m p$ \\
6. safety.mp \\
7. 4 or 5 or 6 \\
8. 3 and 7 \\
\hline
\end{tabular}

were discussed and resolved by mutual consensus. The Newcastle-Ottawa quality assessment scale was used to appraise the quality of case-control studies and the outcomes of interest in cohort studies.

\section{Data abstraction}

A structured data collection form was adopted to compile information from each study including title, year of the study, name of the first author, publication year, country where the study was conducted, demographic and characteristic data of study subjects, exposure measurement, methods used to identify atrial fibrillation, definitions of side effects, and diagnostic methods.

\section{Statistical analysis}

Analyses were performed using the R version 3.5.3 for Mac OS X software program (The R Foundation for Statistical Computing, Vienna, Austria) and the Comprehensive Meta-analysis version 3.3 software program (Biostat Inc., Englewood, NJ, USA). Adjusted point estimates from each study were consolidated by the generic inverse variance approach of DerSimonian and Laird, which determined the weight of each study according to its variance. Given the likelihood of increased interobservation variance, a random-effects model was adopted to assess the pooled prevalence with $95 \%$ CIs for the incidence rates of significant side effects caused by low-dose amiodarone treatment. Cochran's $Q$ test and the $\mathrm{I}^{2}$ statistic were applied to determine the between-study heterogeneity. $\mathrm{An} \mathrm{I}^{2}$ value of $0 \%$ to $25 \%$ represents insignificant heterogeneity, that of $26 \%$ to $50 \%$ represents low heterogeneity, that $51 \%$ of $75 \%$ represents moderate heterogeneity, and that of greater than $75 \%$ represents high heterogeneity. The presence of publication bias was evaluated via the Egger test.

\section{Results}

A total of 2,312 potentially eligible articles were identified using our search strategy. After the exclusion of 2,283 articles, which included case reports, correspondences, review articles, in vitro studies, animal studies, and interventional studies, 29 articles were left for further full-length review. Nineteen studies did not report outcomes of interest and were excluded. Thus, the final analysis included 10 observational studies-specifically, seven retrospective cohort studies $^{7-13}$ and three prospective cohort studies ${ }^{4,14,15}$-involving a total of 901 patients. The literature retrieval, review, and selection processes are shown in Figure 1. The characteristics and quality assessment of the included studies are presented in Table 2.

\section{Definition of amiodarone and its side effects}

We arbitrarily defined low-dose amiodarone as an average maintenance dose of $200 \mathrm{mg}$ /day or less. Significant side effects of amiodarone include those that require medication discontinuation due to the failure of conservative 


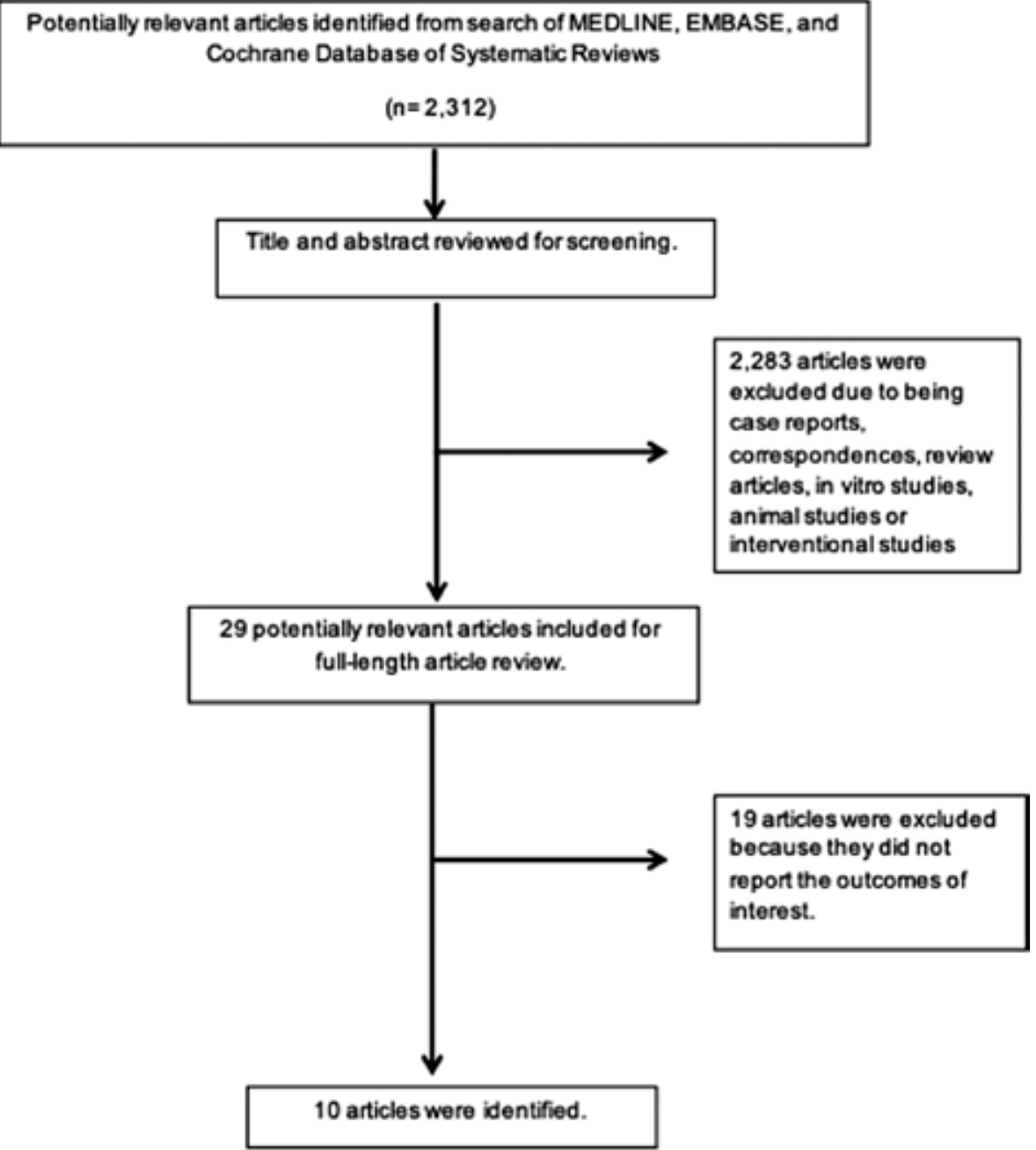

Figure 1: Outline of the search methodology.

treatment of primary organ involvement; hepatotoxicity manifested by a clinically significant transaminitis; significant thyroid disorder diagnosed by laboratory testing along with the clinical manifestation of hyperthyroidism or hypothyroidism ${ }^{16}$; and pulmonary toxicity confirmed by clinical presentation and decreased DLCO without acute heart failure or fibrosis seen on high-resolution pulmonary computed tomography. ${ }^{2}$

Most of the studies (7/10) defined amiodarone-related side effects based on the investigation of clinical presentation and laboratory results.,7-9,11,13,15 Some $(3 / 10)$ reported side effects without providing diagnostic criteria. ${ }^{10,12,14}$ As such, most of the included studies used standard diagnostic tools for establishing the diagnosis of amiodarone-related side effects.

\section{Incidence of significant side effects in patients with amiodarone treatment}

The pooled estimated incidence of overall side effects associated with low-dose amiodarone among the 10 studies included was 17\% (95\% CI: 12\%-22\%; $\mathrm{I}^{2}=73 \%$ )
(Figure 2). However, the pooled estimated incidence of a side effect requiring medication discontinuation was 6\% (95\% CI: 3\%-11\%; $\mathrm{I}^{2}=74.9 \%$ ) (Figure 3). Compared to $200 \mathrm{mg}$ / day of amiodarone, the pooled estimated incidence of overall side effects with a dose of $100 \mathrm{mg} /$ day of amiodarone was 11\% (95\% CI: 4\%-27\%; I²= 80\%) (Figure 4), while the pooled estimated incidence of side effects requiring medication discontinuation was 2\% (95\% CI: $1 \%-6 \% ; \mathrm{I}^{2}=0 \%$ ) (Figure 5$)^{4,15}$ None of the included studies reported any instances of amiodarone-related mortality.

\section{Evaluation for publication bias}

Egger's regression asymmetry test was performed and indicated a presence of publication bias with $p<0.001$ for the incidence of significant side effects associated with low-dose amiodarone treatment.

\section{Discussion}

Our study demonstrates a lower incidence of side effects from low-dose amiodarone. The availability of catheter 


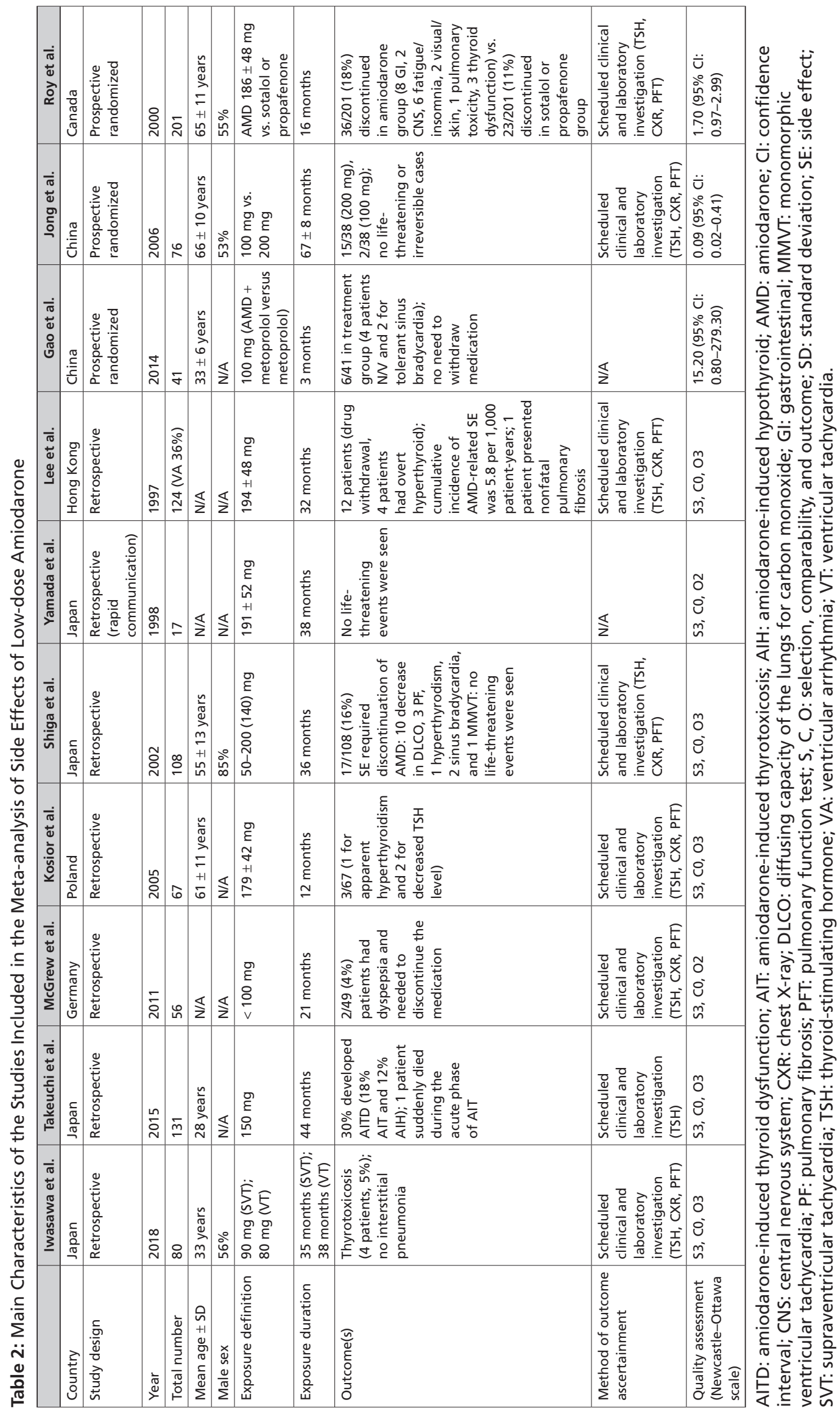




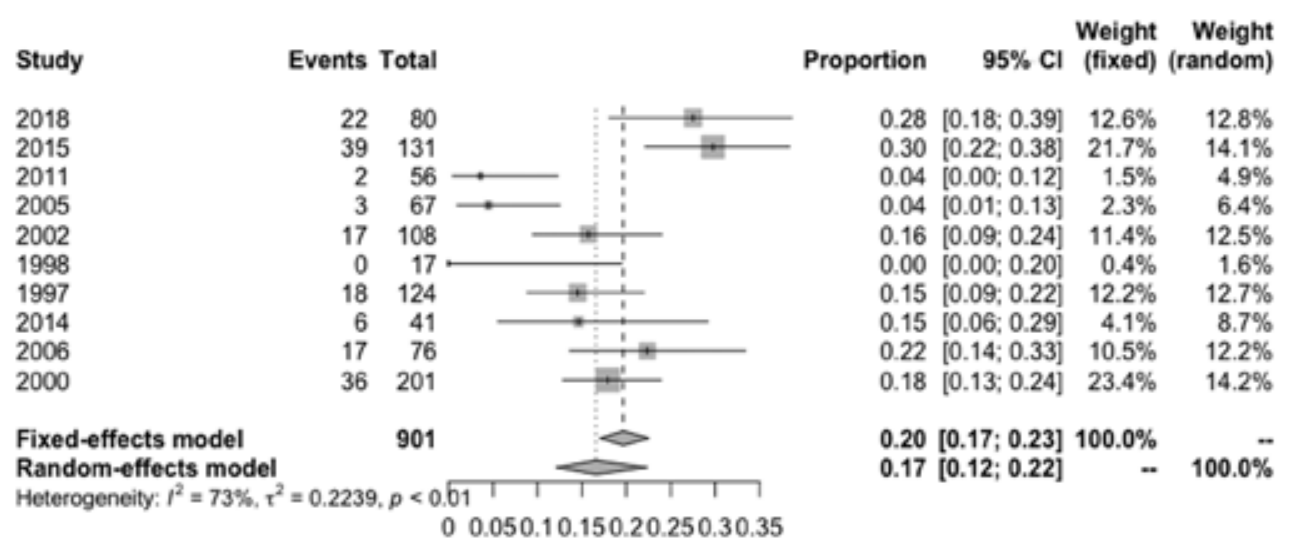

Figure 2: Forest plots of the included studies assessing the incidence of overall side effects of low-dose amiodarone.

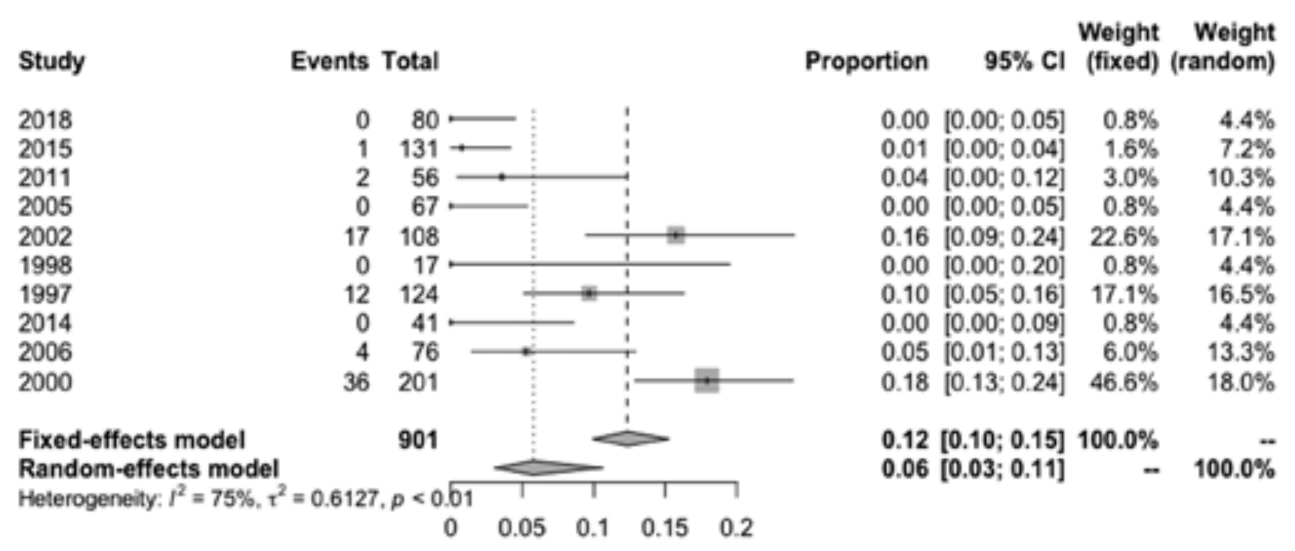

Figure 3: Forest plots of the included studies assessing incidence of serious adverse effects of low-dose amiodarone.

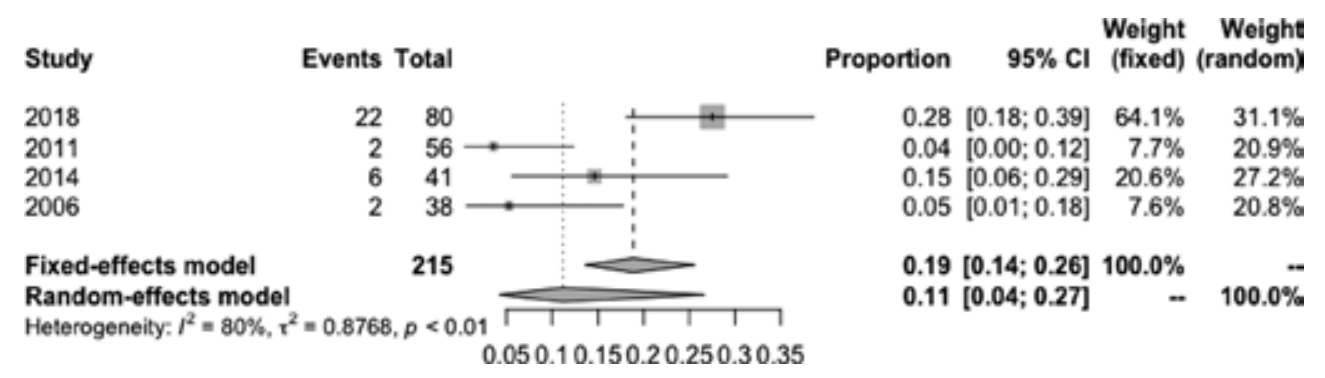

Figure 4: Forest plots of the included studies assessing the incidence of overall side effects of very-low-dose amiodarone.

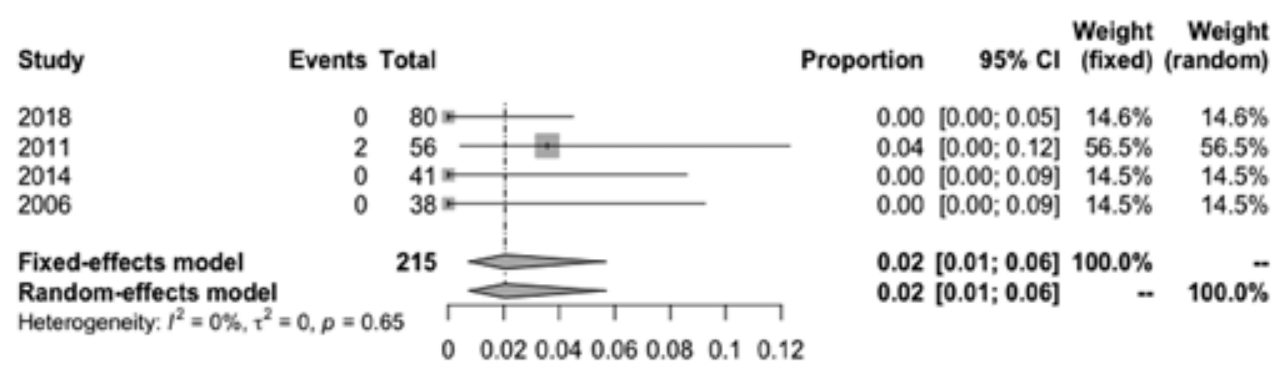

Figure 5: Forest plots of the included studies assessing the incidence of serious adverse effects of very-low-dose amiodarone. 
ablation, ICDs, and other AADs have relegated amiodarone to positioning as a second-line therapy and, when it is used, a lower dosage is dispensed as compared with that used in the past, whereas the therapeutic effects are preserved. To our knowledge, our study is the first meta-analysis to investigate the side effects of "low"-dose amiodarone commonly used in current clinical practice. The incidence of side effects requiring medication discontinuation is estimated to be $4 \%$, which is just one-quarter of that reported by Vorperian et al. ${ }^{3}$ Moreover, none of the surveyed studies demonstrated mortality and many only rarely reported irreversible side effects due to amiodarone administration.

Amiodarone is a well-known medication widely used to treat both supraventricular and ventricular arrhythmias. ${ }^{17}$ Moreover, it is the most efficient drug available for maintaining sinus rhythm in patients with atrial fibrillation. ${ }^{18}$ However, its usage is sometimes limited or even entirely prohibited by side effects, including but not limited to thyroid dysfunction, hepatotoxicity, neurotoxicity, and pulmonary toxicity. Based on a pharmacokinetics study, $100 \mathrm{mg} /$ day of amiodarone can similarly suppress nonsustained ventricular tachycardia as compared with the lowest effective dose of $50 \mathrm{mg} /$ day. ${ }^{19}$ Therefore, the lowest effective dose scientifically confirmed by prior research can still provide a therapeutic effect with minimal intolerable side effects.

It is crucial to understand amiodarone's pharmacokinetics to be able to scrutinize its side effects and toxicity. Due to slow and incomplete gastrointestinal absorption, amiodarone bioavailability varies significantly among different individuals following oral administration. ${ }^{20}$ The amiodarone metabolism by cytochrome P450 3A4 (CYP3A4) in the intestinal wall and gastrointestinal excretion mediated by P-glycoprotein might be major contributors to the overall poor oral bioavailability. ${ }^{21}$ Amiodarone has a high binding affinity to plasma proteins; however, the free fraction is not dependent on either total drug concentration or albumin level..$^{22}$ The exceptionally large volume of distribution of amiodarone is attributable to its high hydrophobicity. ${ }^{23}$ Despite its high fat solubility, the amiodarone plasma concentration might fall by $25 \%$ after a few days of drug cessation even after a steady state is achieved. ${ }^{24}$ Likewise, an animal study indicates that obesity might influence the biodistribution and metabolism of amiodarone. ${ }^{25}$ Amiodarone is mainly metabolized by CYP3A4 to desethylamiodarone and subsequent metabolites, then undergoes biliary excretion, with less than $1 \%$ being excreted in the urine in an unchanged form. ${ }^{26}$

The dosage and duration of amiodarone are the most important factors influencing the risk of developing side effects. Prior to the era of amiodarone-related lethal side effects, the appropriate maintenance dosing to treat significant arrhythmias ranged between $200 \mathrm{mg}$ /day and $800 \mathrm{mg} /$ day for months to years. ${ }^{3,16,27-39}$ After studies showed that side effects were dosing-related, lower dosing, which is $100 \mathrm{mg} /$ day to $200 \mathrm{mg} /$ day, was adopted, thus achieving a better balance between the acceptable side effects and satisfactory treatment outcomes. ${ }^{4,14}$ The lowest dosing of amiodarone was reported by Jong et al. in 2006 in a study designed to investigate the efficacy of low-dose amiodarone on sinus rhythm maintenance after atrial fibrillation cardioversion. ${ }^{4}$ It is noteworthy that no patients in the lower-dose group had significant side effects requiring drug discontinuation. Hence, these safety data encourage the usage of very-low-dose amiodarone.

Regarding side effects, the lack of widely accepted diagnostic criteria and symptom definitions makes it challenging to report the actual incidence of these side effects. Ranging from subclinical to overt clinical manifestations, most of the reported side effects are incidentally revealed through routine laboratory screenings. ${ }^{40}$ Some life-threatening side effects have been reported in patients only on short-term amiodarone, although these extreme adverse events are very rare. ${ }^{41}$ Furthermore, many well-designed studies show that amiodarone is safe under vigilant surveillance. Systematic screening and regular follow-up are the essential elements of these studies. For example, optical coherence tomography is used to detect an early change in retinal fiber layers, which could be reversed by the discontinuation of amiodarone..$^{33}$ Meanwhile, screening and surveillance pulmonary function testing conducted in some studies failed to achieve any benefit in avoiding pulmonary toxicities. ${ }^{36}$ As a result, the available data to clarify the side effects and propose preventive strategies remain under discussion. Moreover, most side effects are diagnosed only after the exclusion of other causes, which could lead to an altered estimation of their incidence rates.

In the last decade, new technologies have been introduced to treat patients with heart diseases who present with concomitant arrhythmias, leading to dramatically decreased morbidity and mortality rates. These new modalities consist of ICDs, cardiac resynchronization therapy systems, and neurohormonal inhibitor medications, which also help to prevent arrhythmias by themselves. ${ }^{42}$ Strategies like these that remain in continued development could further lower the effective dose of amiodarone to control arrhythmia-related problems. Lower doses and improved surveillance would, in theory, result in fewer side effects and improvements in quality of life.

Amiodarone remains the most widely used AAD worldwide. In developing countries, amiodarone is primarily used for atrial fibrillation rate control, whereas, in many developed countries, this drug as a rhythm-control strategy is the mainstream treatment option for qualified patients. The major rate-control drugs are digoxin and amiodarone, which account for $80 \%$ of the medication regimens prescribed by physicians from developing countries. These reflect the popularity of amiodarone, which has been used commonly in many parts of the world. ${ }^{35}$ Blackman et al. previously conducted a survey in European countries about amiodarone use among physicians and found that $100 \mathrm{mg} /$ day of amiodarone is 
widely used by cardiologists in the United Kingdom who believe that "very-low-dose amiodarone" could effectively maintain sinus rhythm in patients with paroxysmal atrial fibrillation. ${ }^{6}$

There are some limitations in our study. First, this study was a meta-analysis of observational studies; hence, we cannot draw a conclusion regarding a causal relationship from the results. Also, the common inherent biases in this study design are recall bias and observational bias. Second, the caveat of data interpretation is the heterogeneity of establishing a side effect diagnosis based on different diagnostic tools and criteria. However, we at least demonstrated that actual serious side effects requiring medication cessation do not commonly appear.

In conclusion, our study demonstrates the safety of verylow-dose amiodarone, which has been prescribed worldwide. In addition, the incidence of side effects from our study is lower than that of a prior meta-analysis, which might encourage physicians to begin or continue to use this useful medication, albeit with limitations as appropriate. Further research should be conducted to provide stronger evidence regarding the relationship between low-dose amiodarone and related side effects.

\section{References}

1. Zimetbaum P. Antiarrhythmic drug therapy for atrial fibrillation. Circulation. 2012;125(2):381-389.

2. Epstein AE, Olshansky B, Naccarelli GV, Kennedy JI Jr, Murphy EJ, Goldschlager N. Practical management guide for clinicians who treat patients with amiodarone. Am J Med. 2016;129(5):468-475.

3. Vorperian VR, Havighurst TC, Miller S, January CT. Adverse effects of low dose amiodarone: a meta-analysis. J Am Coll Cardiol. 1997;30(3):791-798.

4. Jong GP, Chang MH, Chang TC, et al. Long-term efficacy and safety of very-low-dose amiodarone treatment for the maintenance of sinus rhythm in patients with chronic atrial fibrillation after successful direct-current cardioversion. Chin Med J (Engl). 2006;119(24):2030-2035.

5. Kochiadakis GE, Igoumenidis NE, Hamilos MI, et al. Longterm maintenance of normal sinus rhythm in patients with current symptomatic atrial fibrillation: amiodarone vs propafenone, both in low doses. Chest. 2004;125(2):377-383.

6. Blackman DJ, Ferguson JD, Bashir Y. Dose of amiodarone in atrial fibrillation-neither guidelines nor clinical practice reflect available evidence. Eur Heart J. 2002;23(11):908.

7. Kosior DA, Wozakowska-Kaplon B, Jasik M, Kiliszek M, Rabczenko D, Opolski G. Amiodarone after unsuccessful direct-current cardioversion of persistent atrial fibrillation. Kardiol Pol. 2005;63(6):585-592; discussion 593-594.

8. Iwasawa S, Uyeda T, Saito M, et al. Efficacy and safety of low-dose amiodarone therapy for tachyarrhythmia in congenital heart disease. Pediatr Cardiol. 2018.

9. Takeuchi D, Honda K, Shinohara T, Inai K, Toyohara K, Nakanishi T. Incidence, clinical course, and risk factors of amiodarone-induced thyroid dysfunction in Japanese adults with congenital heart disease. Circ J. 2015;79(8):1828-1834.

10. McGrew FA, Foon J, Charlton SB. Extremely low dose amiodarone is a safe and effective strategy in AF management. J Card Fail. 2012;18(8):S101.
11. Shiga T, Wakaumi M, Imai T, et al. Effect of low-dose amiodarone on atrial fibrillation or flutter in Japanese patients with heart failure. Circ J. 2002;66(6):600-604.

12. Yamada S, Yamaguchi I. Effect of relatively low dose amiodarone therapy on left ventricular function in patients with ventricular tachyarrhythmias. Jpn Circ J. 1998;62(9): 707-709.

13. Lee KL, Tai YT. Long-term low-dose amiodarone therapy in the management of ventricular and supraventricular tachyarrhythmias: efficacy and safety. Clin Cardiol. 1997;20(4):372-377.

14. Gao Y, Zhang P, Liang X. Effects of low-dose amiodarone and Betaloc on the treatment of hypertrophic cardiomyopathy complicated with malignant ventricular arrhythmias. Pak J Med Sci. 2014;30(2):291-294.

15. Roy D, Talajic M, Dorian $\mathrm{P}$, et al. Amiodarone to prevent recurrence of atrial fibrillation. Canadian Trial of Atrial Fibrillation Investigators. N Engl J Med. 2000;342(13):913-920.

16. Bouvy ML, Heerdink ER, Hoes AW, Leufkens HG. Amiodarone-induced thyroid dysfunction associated with cumulative dose. Pharmacoepidemiol Drug Saf. 2002;11(7):601-606.

17. Zimetbaum P. Amiodarone for atrial fibrillation. $N$ Engl J Med. 2007;356(9):935-941.

18. Razavi M. Safe and effective pharmacologic management of arrhythmias. Texas Heart Inst J. 2005;32(2):209-211.

19. Mahmarian JJ, Smart FW, Moye LA, et al. Exploring the minimal dose of amiodarone with antiarrhythmic and hemodynamic activity. Am J Cardiol. 1994;74(7):681-686.

20. Andreasen F, Agerbaek H, Bjerregaard P, Gotzsche H. Pharmacokinetics of amiodarone after intravenous and oral administration. Eur J Clin Pharmacol. 1981;19(4):293-299.

21. KashimaA,FunahashiM,FukumotoK, etal.Pharmacokinetic characteristics of amiodarone in long-term oral therapy in Japanese population. Biol Pharm Bull. 2005;28(10):1934-1938.

22. Veronese ME, McLean S, Hendriks R. Plasma protein binding of amiodarone in a patient population: measurement by erythrocyte partitioning and a novel glass-binding method. Br J Clin Pharmacol. 1988;26(6):721-731.

23. Holt DW, Tucker GT, Jackson PR, Storey GC. Amiodarone pharmacokinetics. Am Heart J. 1983;106(4 Pt 2):840-847.

24. Pollak PT, Bouillon T, Shafer SL. Population pharmacokinetics of long-term oral amiodarone therapy. Clin Pharmacol Ther. 2000;67(6):642-652.

25. Abdussalam A, Al-Agili M, Al Nebaihi HM, Mayo PR, Gabr RQ, Brocks DR. Dietary-induced obesity and changes in the biodistribution and metabolism of amiodarone in the rat. $J$ Pharm Sci. 2018;107(11):2938-2945.

26. Fukumoto K, Kobayashi T, Tachibana K, et al. Effect of amiodarone on the serum concentration/dose ratio of metoprolol in patients with cardiac arrhythmia. Drug Metab Pharmacokinet. 2006;21(6):501-505.

27. Arkun A, Van Deusen SK, Grau T, Birkhahn RH. Hepatic dysfunction and neurotoxicity in a patient receiving long-term low-dose amiodarone therapy. J Emerg Med. 2010;38(3):337-339.

28. Brennan FJ, Brien JF, Armstrong PW. Plasma concentration time course and pharmacological effects of a standardized oral amiodarone dosing regimen in humans. Can J Cardiol. 1991;7(3):117-124.

29. Gefter WB, Epstein DM, Pietra GG, Miller WT. Lung disease caused by amiodarone, a new antiarrythmic agent. Radiology. 1983;147(2):339-344.

30. Greene HL, Graham EL, Werner JA, et al. Toxic and therapeutic effects of amiodarone in the treatment of cardiac arrhythmias. J Am Coll Cardiol. 1983;2(6):1114-1128. 
31. Kennedy JI, Myers JL, Plumb VJ, Fulmer JD. Amiodarone pulmonary toxicity. Clinical, radiologic, and pathologic correlations. Archiv Internal Med. 1987;147(1):50-55.

32. Kervinen M, Falck A, Hurskainen M, Hautala N. Bilateral optic neuropathy and permanent loss of vision after treatment with amiodarone. J Cardiovasc Pharmacol. 2013;62(4):394-396.

33. Martinez-LoPez-Portillo MA, Martinez-Gamero BO, Mohamed-Noriega J, Cavazos-Adame $\mathrm{MH}$, MohamedHamsho MJ. Behaviour of disc oedema during and after amiodarone optic neuropathy: case report. J Clin Diagn Res. 2014;8(4):Vd04-Vd05.

34. Mindel JS, Anderson J, Hellkamp A, et al. Absence of bilateral vision loss from amiodarone: a randomized trial. Am Heart J. 2007;153(5):837-842.

35. Myers JL, Kennedy JI, Plumb VJ. Amiodarone lung: pathologic findings in clinically toxic patients. Hum Pathol. 1987;18(4):349-354.

36. Ohar JA, Jackson F Jr, Redd RM, Evans GR, Bedrossian CW. Usefulness of serial pulmonary function testing as an indicator of amiodarone toxicity. Am J Cardiol. 1989;64(19):1322-1326.

37. O'Sullivan AJ, Lewis M, Diamond T. Amiodarone-induced thyrotoxicosis: left ventricular dysfunction is associated with increased mortality. Eur J Endocrinol. 2006;154(4):533-536.

38. Purvin V, Kawasaki A, Borruat FX. Optic neuropathy in patients using amiodarone. Archiv Ophthalmol (Chicago, Ill: 1960). 2006;124(5):696-701.

39. Rigas B, Rosenfeld LE, Barwick KW, et al. Amiodarone hepatotoxicity. A clinicopathologic study of five patients. Ann Internal Med. 1986;104(3):348-351.

40. Cohen-Lehman J, Dahl P, Danzi S, Klein I. Effects of amiodarone therapy on thyroid function. Nat Rev Endocrinol. 2010;6(1):34-41.

41. Singhal A, Ghosh P, Khan SA. Low dose amiodarone causing pseudo-alcoholic cirrhosis. Age Ageing. 2003;32(2):224-225.

42. Cleland JG, Pellicori P, Dicken B. Why does CRT reduce the risk of arrhythmias? JACC Cardiovasc Imaging. 2013;6(4):445-447. 